Assessment of 70-keV virtual

monoenergetic spectral images in

abdominal CT imaging: A comparison

study to conventional polychromatic 120-

$k V p$ images

Negin Rassouli, Hamid Chalian,

Prabhakar Rajiah, Amar Dhanantwari \& Luis Landeras

Abdominal Radiology

ISSN 2366-004X

Abdom Radiol

DOI 10.1007/s00261-017-1151-2

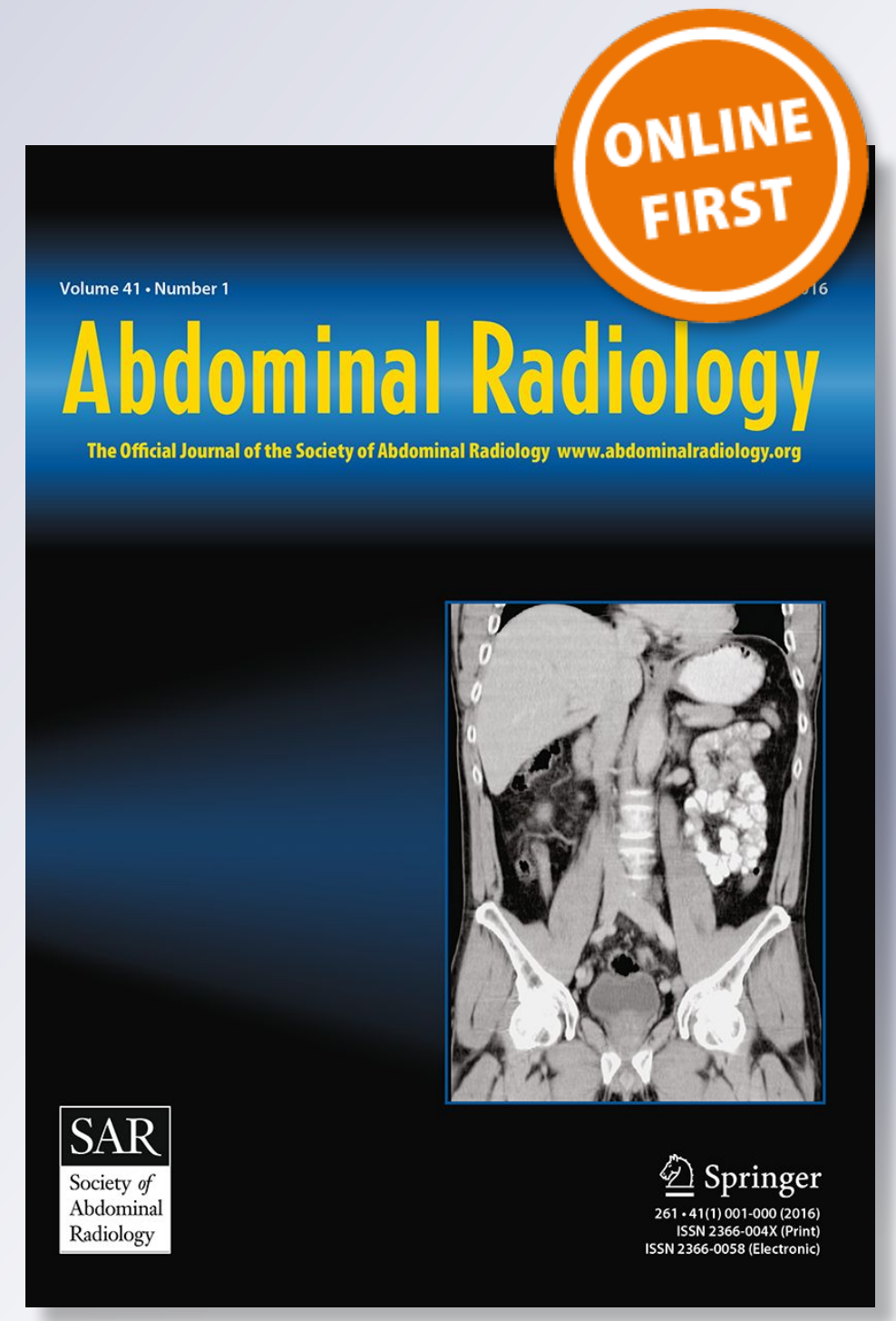

留 Springer 
Your article is protected by copyright and all rights are held exclusively by Springer Science +Business Media New York. This e-offprint is for personal use only and shall not be selfarchived in electronic repositories. If you wish to self-archive your article, please use the accepted manuscript version for posting on your own website. You may further deposit the accepted manuscript version in any repository, provided it is only made publicly available 12 months after official publication or later and provided acknowledgement is given to the original source of publication and a link is inserted to the published article on Springer's website. The link must be accompanied by the following text: "The final publication is available at link.springer.com". 


\title{
Assessment of 70-keV virtual monoenergetic spectral images in abdominal CT imaging: A comparison study to conventional polychromatic 120-kVp images
}

\author{
Negin Rassouli, ${ }^{1}$ Hamid Chalian, ${ }^{1}$ Prabhakar Rajiah, ${ }^{2}$ Amar Dhanantwari, ${ }^{3}$ \\ Luis Landeras ${ }^{4}$
}

${ }^{1}$ Department of Radiology, University Hospitals Cleveland Medical Center, Case Western Reserve University School of Medicine, Cleveland, OH, USA

${ }^{2}$ Department of Radiology, Cardiothoracic Imaging, UT Southwestern Medical Center, Dallas, TX, USA

${ }^{3}$ Philips Healthcare, Cleveland, OH, USA

${ }^{4}$ Department of Radiology, University of Chicago, Chicago, IL, USA

\begin{abstract}
Purpose: To evaluate the image quality of $70-\mathrm{keV}$ virtual monoenergetic (monoE) abdominal CT images compared to $120-\mathrm{kVp}$ polychromatic images generated from a spectral detector CT (SDCT) scanner.

Methods: This prospective study included generation of a $120-\mathrm{kVp}$ polychromatic dataset and a $70-\mathrm{keV}$ virtual monoE dataset after a single contrast-enhanced CT acquisition on a SDCT scanner (Philips Healthcare) during portal venous phase. The attenuation values (HU), noise, signal-to-noise ratio (SNR), and contrastto-noise ratio (CNR) were measured in the liver, spleen, pancreas, kidney, aorta, portal vein, and muscle. The subjective image quality including noise, soft tissue contrast, sharpness, and overall image quality were graded on a 5-point Likert scale by two radiologists independently (1-worst image quality, 5-best image quality). Statistical analysis was performed using paired sample t test and Fleiss's Kappa.

Results: Fifty-five patients $(54.3 \pm 16.8$ y/o; $28 \mathrm{M}, 27 \mathrm{~F})$ were recruited. The noise of target organs was significantly lower in virtual monoE images in comparison to polychromatic images $(p<0.001)$. The SNR and CNR were significantly higher in virtual monoE images $(p<0.001$ for both). Subjective image quality of $70-\mathrm{keV}$ virtual monoE images was significantly better $(p<0.001)$ for all evaluated parameters. Median scores
\end{abstract}

Correspondence to: Negin Rassouli; email: negin_rassouli@yahoo.com for all subjective parameters were 3.0 versus 4.0 for polychromatic vs virtual monoE images, respectively. The inter-reader agreement for overall image quality was good (Kappa were 0.767 and 0.762 for polychromatic and virtual monoE images, respectively). Conclusion: In abdominal imaging, $70-\mathrm{keV}$ virtual monoE CT images demonstrated significantly better noise, SNR, CNR, and subjective score compared to conventional $120-\mathrm{kVp}$ polychromatic images.

Key words: Spectral detector CT - SDCT - Dual-layer CT-Monoenergetic image

Computed tomography (CT) has become an established technology in the clinical care because of its wide availability, low cost compared to MRI, and consistent image quality [1]. In traditional CT, X-ray generated by the $\mathrm{X}$-ray tube has a continuous energy distribution called polychromatic X-ray. Since the introduction of CT, polychromatic $120-\mathrm{kVp} \mathrm{X}$-rays have been widely used in clinical diagnosis as the standard acquisition in abdominal CT imaging [2-6].

In recent years, the emergence of spectral imaging has improved the detection rate of diseases and has offset some issues of the traditional CT imaging including beam hardening artifact and tissue characterization [3]. Currently, three spectral CT scanners 
are available, including (1) CT scanner with 2 X-ray tubes spaced approximately $90^{\circ}$ apart and 2 detector sets manufactured by Siemens Medical Solutions (dual source dual detector), (2) CT Scanner with one rapid $\mathrm{kVp}$-switching source and new detector based on gemstone scintillator materials manufactured by GE Healthcare (single source dual energy), and (3) CT scanner with a single source and two layers of detectors manufactured by Philips Healthcare (single source dual detector) which enables acquisition of conventional $120-\mathrm{kVp}$ CT images and retrospective generation of different spectrums of virtual monoenergetic CT images (spectral detector CT, SDCT). Although there is only a single $\mathrm{X}$-ray source, there are two layers of detector. The top layer selectively absorbs low energy photons and the bottom layer absorbs high energy photons, thus providing two distinct energy datasets. In addition to the conventional $120-\mathrm{kVp}$ images that are obtained by utilizing combined data from both detector layers, additional spectral analysis can be obtained by decomposition of the low and high energy data into photoelectric and Compton data components of attenuation.

The advantages of spectral CT imaging include the ability to generate virtual non-contrast images, which has the potential to decrease radiation and cost, iodine map, effective atomic number $(Z)$, and virtual monoenergetic images at different $\mathrm{keV}$ levels throughout the X-ray spectrum. These on-demand virtual monoE images can give us extra information and can improve the evaluation and characterization of lesions. Virtual monoE images can also reduce different artifact (metal, beam hardening, and blooming) [7-11]. No need for prospective patient selection, no rotation time limitations, full FOV, and no cross scatter are among the unique advantages of SDCT in comparison to the other spectral technologies. However, SDCT has some limitations including short $\mathrm{Z}$ axis $(4 \mathrm{~cm})$ and requirement of at least $120-\mathrm{kVp}$ tube potential.

We compared $70-\mathrm{keV}$ virtual monoE images to $120-\mathrm{kVp}$ polychromatic images generated from a SDCT scanner in this study. The choice of $70-\mathrm{keV}$ was based on previously published reports which showed the lowest image noise and the highest contrast-to-noise ratio at $70-\mathrm{keV}$ among more than hundred sets of virtual monoenergetic images in the range of $40-140 \mathrm{keV}$ (2). Although there are few published articles comparing the spectral CT imaging with the conventional $120-\mathrm{kVp}$ CT imaging, to the best of our knowledge, no clinical study has compared SDCT to conventional $120-\mathrm{kVp}$ CT images.

The purpose of this study was to evaluate the objective and subjective image quality of $70-\mathrm{keV}$ virtual monoE images compared to $120-\mathrm{kVp}$ polychromatic images generated from a SDCT scanner.

\section{Materials and methods}

\section{Study population}

This prospective HIPPA compliant study was approved by the institutional review board of our institution. Written informed consent was obtained from all of the patients. From October 2013 to December 2015, patients older than 18 years who scheduled for contrast-enhanced CT imaging of the abdomen or abdomen and pelvis as part of their clinical standard-of-care study were included. CT scans with suboptimal portal phase (mean attenuation $<70 \mathrm{HU}$ in portal vein), metal artifact, or lack of the upper abdomen were excluded. Fifty-five patients were finally recruited to our study. The scan time (the time patient was in the CT machine) and radiation dose were not affected by using SDCT scanner.

\section{Imaging protocol and reconstruction}

Abdominal examinations were performed for various clinical indications, including pancreas, liver or renal mass evaluation, pre-renal and liver transplant assessment, or abdominal pain evaluation using an SDCT scanner (product name IQon, Philips, Cleveland, $\mathrm{OH}$ ). Post-contrast imaging was performed in the portal venous/nephrographic (65-120 s delay) phase only or multiphasic including the arterial, portal venous/ nephrographic, and excretory (5-10 min delay) phases as indicated clinically. However, for the purpose of this study, image analysis was done on portal venous phase images. Isovue 370 (Bracco Diagnostics Inc, Princeton, NJ) or Ultravist 350 (Bayer HealthCare, Wayne, NJ) was used as contrast and the dose varied depending on the protocol and weight. All examinations were performed at a tube potential of $120-\mathrm{kVp}$ and a detector configuration of $64 \times 0.625 \mathrm{~mm}$ with automatic tube current modulation. Scans were performed at pitch range of 0.5-1.17 and gantry rotation time of $0.3-0.75 \mathrm{~s}$ depending on the clinical protocol. All examinations were performed in the supine position. Slice thickness was $2 \mathrm{~mm}$ at $1 \mathrm{~mm}$ increments. On the SDCT, images were obtained directly from the scanner which combined the data from the top and bottom detector layers and utilized iterative reconstruction algorithm (iDose ${ }^{4}$ Level 3). $70-\mathrm{keV}$ virtual monoE images were reconstructed retrospectively using the spectral diagnostic suite (SPDS, Philips, Haifa, Israel).

\section{Image analysis}

Image analysis was performed retrospectively. Conventional $120-\mathrm{kVp}$ and $70-\mathrm{keV}$ virtual monoE images were sent to a separate viewing station (SPDS, Philips, Haifa, Israel). Conventional $120-\mathrm{kVp}$ and $70-\mathrm{keV}$ virtual monoE post-contrast images were evaluated side by side 


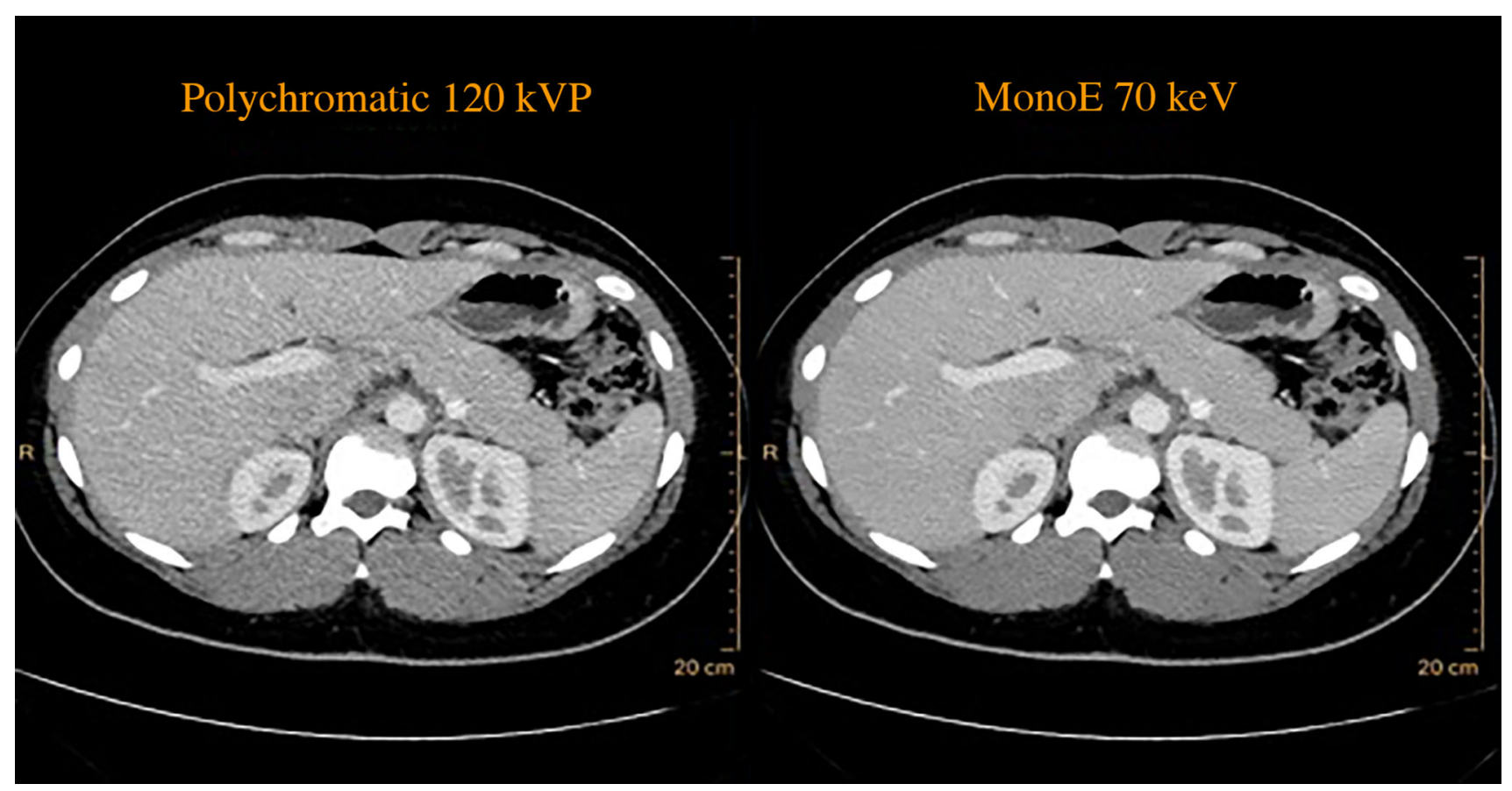

Fig. 1. Polychromatic $120-k V p$ vs. $70-k e V$ virtual monoE images. Significantly better overall image quality, noise, sharpness, and tissue contrast was observed in our study for virtual monoE images as compared to the conventional 120-kVp polychromatic images.

Table 1. Subjective scoring of image quality using 5-point Likert scale

\begin{tabular}{lllll}
\hline & Image noise & Soft tissue contrast & Sharpness & Overall image quality \\
\hline 5 & Minimum/no noise & Excellent & Sharpest & Best \\
4 & Less than average & Above average & Above average & Above average \\
3 & Average & Average & Average & Average \\
2 & Above average & Below average & Below average & Below average \\
1 & Unacceptable & Poor & Blurry & Unacceptable \\
\hline
\end{tabular}

for objective analysis (Fig. 1). Multiple regions of interest (ROI) were drawn by one of the two readers in liver (segment 1), spleen, pancreas, portal vein, renal cortex, abdominal aorta, paraspinal muscle, and abdominal fat in the posterior abdominal wall (as background) on conventional $120-\mathrm{kVp}$ images. The ROI was then copied to the $70-\mathrm{keV}$ virtual monoE images to ensure constant size and location of the ROI. The size of each ROI was approximately $20 \mathrm{~mm}$. Smaller size was used when 20 $\mathrm{mm}$ ROI placement was not possible.

\section{Objective image quality}

CT attenuation values (Hounsfield units [HU]) and image noise [standard deviation (SD)] were recorded for all image sets at different locations. The signal-to-noise ratio (SNR) was then calculated by dividing the CT attenuation values by the corresponding image noise. The contrast-to-noise ratio (CNR) of the liver, kidney, pancreas, spleen, portal vein, and aorta were calculated using the following formulae [2, 12-14]:
$\mathrm{CNR}_{\text {Liver,Kidney,Pancreas,Spleen }}$

$=\left(\mathrm{ROI}_{\text {Liver,Kidney,Pancreas,Spleen }}-\mathrm{ROI}_{\text {Background }}\right) / \mathrm{SD}_{\text {Background }}$

$\mathrm{CNR}_{\text {Portalvein }}=\left(\mathrm{ROI}_{\text {Portalvein }}-\mathrm{ROI}_{\text {Liver }}\right) / \mathrm{SD}_{\text {Background }}$

$\mathrm{CNR}_{\text {Aorta }}=\left(\mathrm{ROI}_{\text {Aorta }}-\mathrm{ROI}_{\text {Muscle }}\right) / \mathrm{SD}_{\text {Background }} \cdot$

ROI in multiple organs denote the attenuation values (HU) of these organs. SD and ROI background denotes the image noise and attenuation of fat, respectively [2, 12-14].

\section{Subjective image quality}

Subjective image quality of each conventional $120-\mathrm{kVp}$ and $70-\mathrm{keV}$ virtual monoE image was assessed by two blinded radiologists with 6 and 12 years of experience in abdominal imaging independently. The image quality, including noise, soft tissue contrast, sharpness (with re- 


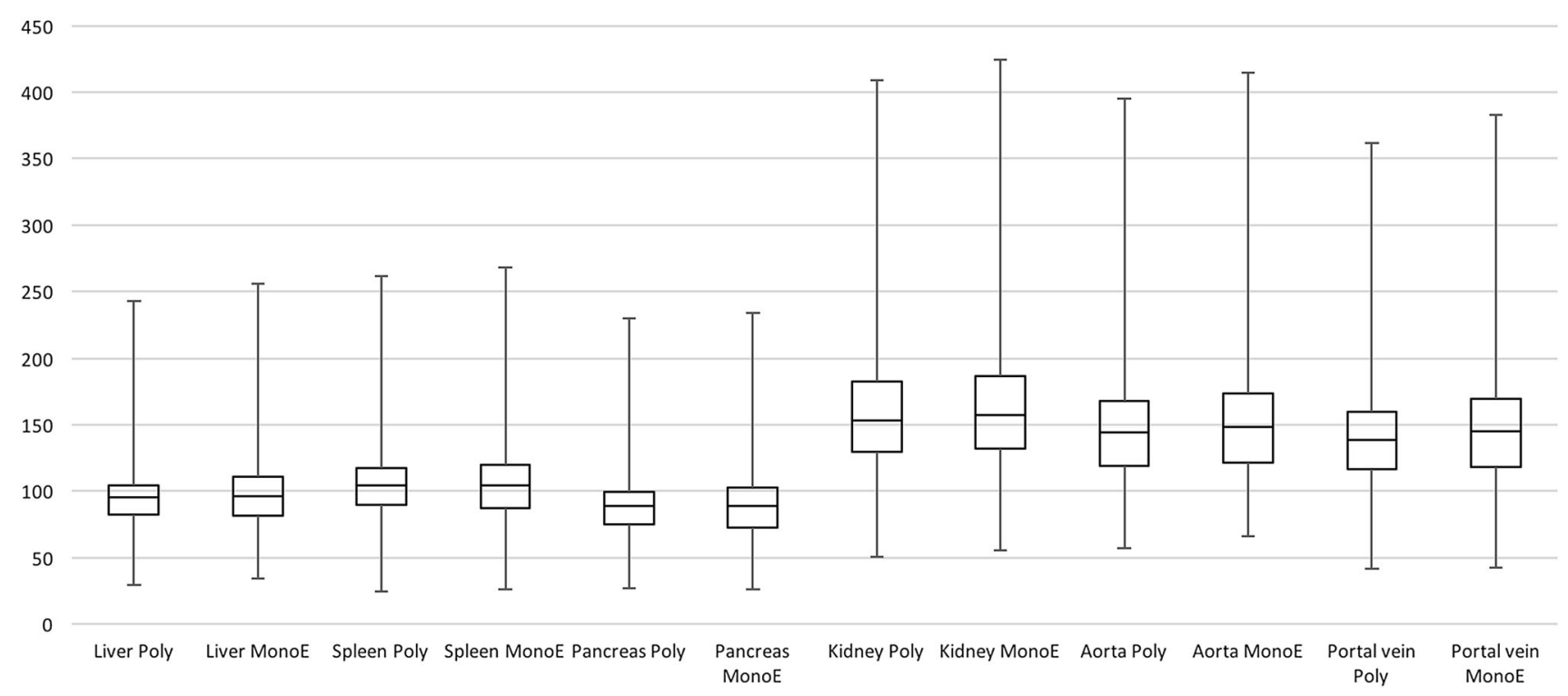

Fig. 2. Mean attenuation (HU) in conventional 120-kVp polychromatic images in comparison to $70-\mathrm{keV}$ virtual monoE images. Kidney, aorta, and portal vein demonstrated significantly higher CT attenuation in virtual monoE images as compared to conventional $120-\mathrm{kVp}$ images $(p<0.05)$.
CT attenuation of the liver, spleen, and pancreas was comparable between virtual monoE images and conventional $120-\mathrm{kVp}$ images $(p>0.05)$. Box shows 25th and 75th percentiles. Error bars demonstrated 2 standard deviations from mean.

Table 2. Comparison of CT attenuation and noise between $70-\mathrm{keV}$ virtual monoE images and conventional $120-\mathrm{kVp}$ polychromatic images

\begin{tabular}{|c|c|c|c|c|c|c|}
\hline \multirow[t]{2}{*}{ Organ } & \multicolumn{3}{|c|}{ Attenuation value (HU) } & \multicolumn{3}{|c|}{ Noise (SD) } \\
\hline & Poly & monoE & $p$ value & Poly & monoE & $p$ value \\
\hline Liver & $95.14 \pm 21.42$ & $96.30 \pm 24.18$ & 0.173 & $18.23 \pm 4.89$ & $14.64 \pm 3.68$ & $<0.001$ \\
\hline Spleen & $104.68 \pm 19.63$ & $104.72 \pm 21.94$ & 0.954 & $15.68 \pm 5.09$ & $12.55 \pm 4.18$ & $<0.001$ \\
\hline Pancreas & $88.69 \pm 20.67$ & $88.61 \pm 21.22$ & 0.840 & $16.86 \pm 4.04$ & $13.88 \pm 3.42$ & $<0.001$ \\
\hline Kidney & $153.06 \pm 36.78$ & $157.10 \pm 40.39$ & 0.001 & $17.49 \pm 5.42$ & $14.77 \pm 5.30$ & $<0.001$ \\
\hline Aorta & $144.50 \pm 41.34$ & $148.75 \pm 46.35$ & 0.028 & $19.10 \pm 5.04$ & $15.38 \pm 4.17$ & $<0.001$ \\
\hline Portal vein & $138.51 \pm 31.77$ & $144.84 \pm 34.25$ & $<0.001$ & $18.34 \pm 5.00$ & $15.42 \pm 4.70$ & $<0.001$ \\
\hline
\end{tabular}

spect to the borders of organs and intrahepatic vascular wall), and overall image quality were graded on a 5-point Likert scale (1-worst image quality, 5-best image quality, $\leq 3$ defined as fair, and $>3$ defined as good) (Table 1).

\section{Statistical analysis}

Statistical analysis was performed using SPSS version 21.0 (IBM Corp, 2012). Quantitative variables were expressed as mean \pm standard deviation (SD). Paired $t$ test was used to compare the attenuation value (HU), objective image noise, SNRs, and CNRs among the polychromatic and $70-\mathrm{keV}$ virtual monoE images. A $p$ value of $<0.05$ was considered statistically significant. Fleiss's Kappa and McNemar's test were used to evaluate the agreement between the two readers for the subjective image quality scoring. Poor reliability was defined as a kappa of $<0.4$, fair reliability as $0.4-0.6$, good reliability as $>0.6-0.8$, and excellent reliability as $>0.8$.

\section{Results}

Fifty-five patients ( 28 males, 27 females) with the mean age of $54.3 \pm 16.9$ years old were recruited in our study.

The objective image quality measurements including the CT attenuation (HU), noise (SD), SNR, and CNR in standard $120-\mathrm{kVp}$ polychromatic images versus $70-\mathrm{keV}$ virtual monoE images are summarized in Tables 2 and 3.

At $70-\mathrm{keV}$ virtual monoE level, there was no significant difference in CT attenuation of the liver, spleen, and pancreas but attenuation of the kidney, aorta, and portal vein was significantly higher $(p<0.05)$ in virtual monoE images in comparison to polychromatic images (Fig. 2). The noise of target organs was significantly lower $(p<0.001)$ in virtual monoE images as compared to polychromatic images (Table 2; Fig. 3). 


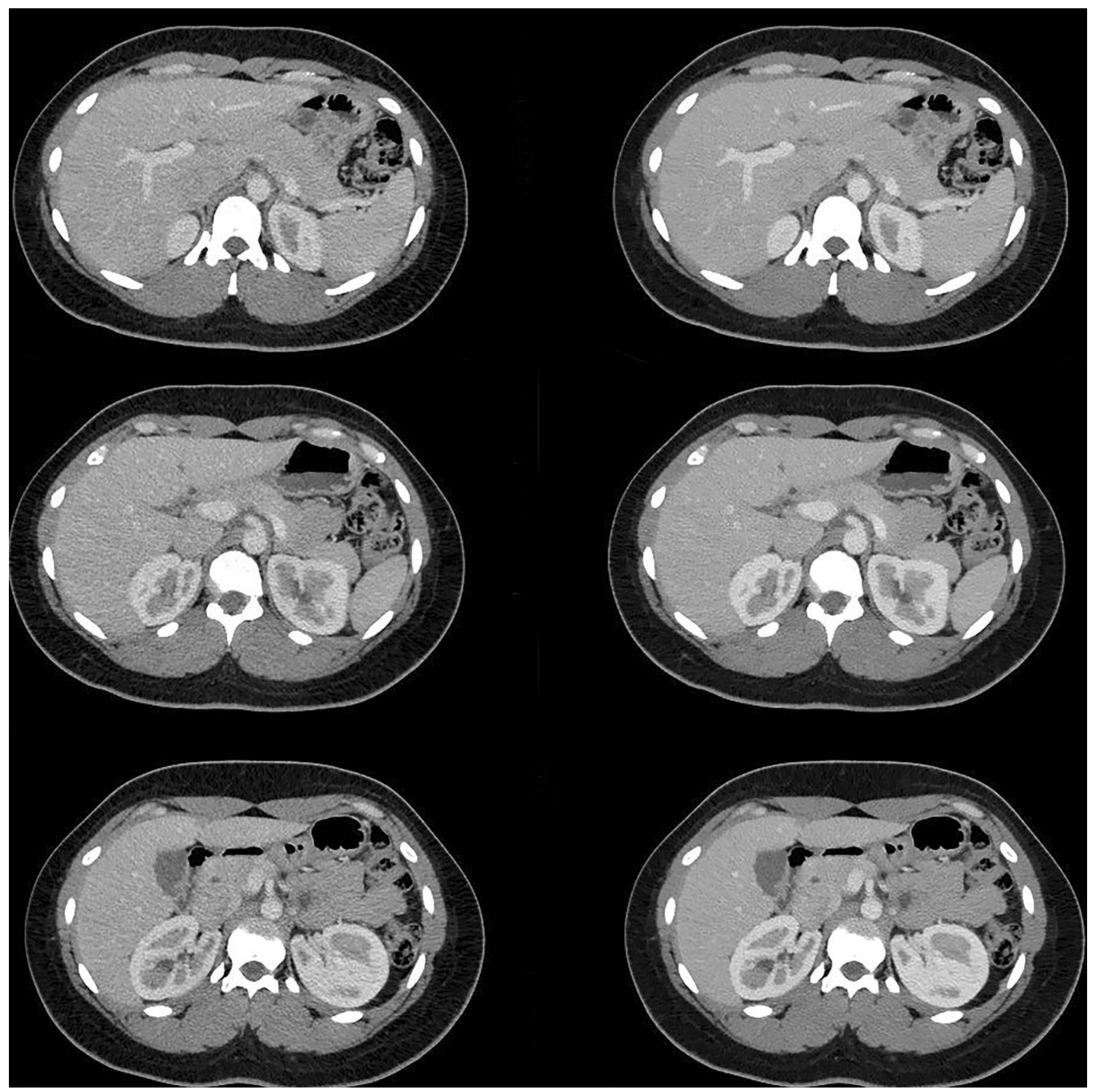

Fig. 3. Transverse abdominal CT images obtained in a 28-year-old woman. The noise is higher in polychromatic images (left) in comparison to virtual monoE images (right). The Noise in the liver was 12.3 in conventional $120-k V p$ polychromatic images and 9.8 in virtual monoE images. The SNR and CNR are higher in virtual monoE images (right) in

The SNR of target organs was significantly higher $(p<0.001)$ in virtual monoE images (Fig. 3). The CNR of the target organs was also significantly higher $(p<0.001)$ in virtual monoE images (Table 3; Fig. 3).

Subjective image quality of $70-\mathrm{keV}$ virtual monoE images was significantly better $(p<0.001)$ for all evaluated parameters, including noise, soft tissue contrast, comparison to polychromatic images (left). The SNR in the spleen was 9.61 in conventional $120-\mathrm{kVp}$ polychromatic images and 12.34 in virtual monoE images. The CNR in the spleen was 18.70 in conventional $120-k V p$ polychromatic images and 24.52 in virtual monoE images.

sharpness, and overall image quality (Fig. 4). Median scores (poly vs. virtual monoE) were 3.0 and 4.0 for all subjective parameters (Table 4).

The inter-observer agreement for all evaluated parameters was good and Kappa values for polychromatic and virtual monoE images are summarized in Table 5 . 


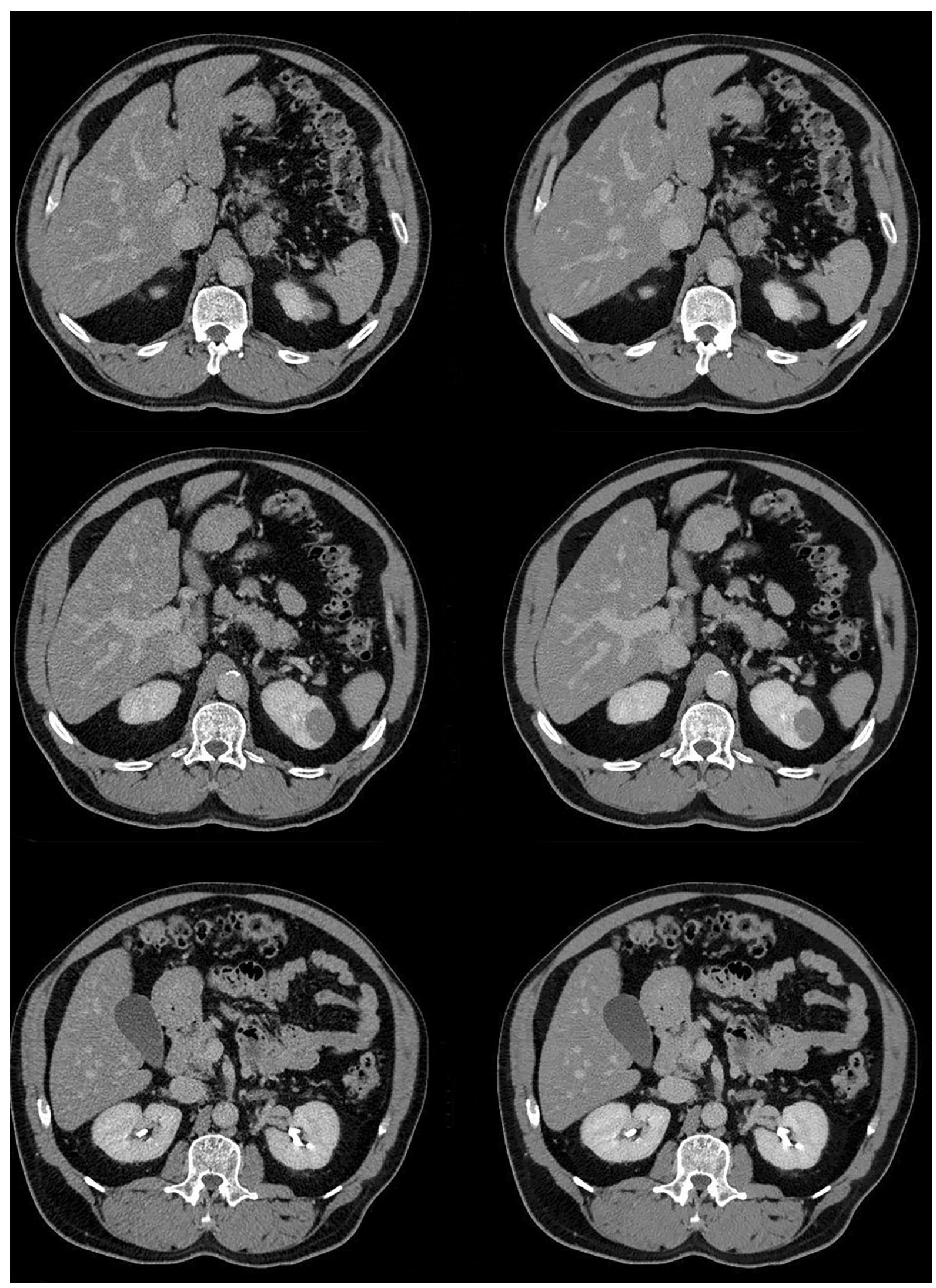

Fig. 4. Axial CT images of the abdomen obtained in an 87-year-old man. 70-keV virtual monoE images (right) had better subjective scores in comparison to polychromatic $120-k V p$ images (left). 
Table 3. Comparison of SNR and CNR between $70-\mathrm{keV}$ virtual monoE images and conventional $120-\mathrm{kVp}$ polychromatic images

\begin{tabular}{|c|c|c|c|c|c|c|}
\hline \multirow[t]{2}{*}{ Organ } & \multicolumn{3}{|c|}{ SNR } & \multicolumn{3}{|c|}{ CNR } \\
\hline & Poly & monoE & $p$ value & Poly & monoE & $p$ value \\
\hline Liver & $5.84 \pm 2.01$ & $6.99 \pm 2.63$ & $<0.001$ & $19.33 \pm 5.89$ & $24.29 \pm 7.17$ & $<0.001$ \\
\hline Spleen & $7.31 \pm 2.50$ & $9.17 \pm 3.46$ & $<0.001$ & $20.05 \pm 5.78$ & $25.10 \pm 7.16$ & $<0.001$ \\
\hline Pancreas & $5.66 \pm 2.23$ & $6.91 \pm 2.79$ & $<0.001$ & $18.71 \pm 5.72$ & $23.34 \pm 6.87$ & $<0.001$ \\
\hline Kidney & $9.55 \pm 3.91$ & $11.72 \pm 4.62$ & $<0.001$ & $24.84 \pm 7.77$ & $31.62 \pm 9.76$ & $<0.001$ \\
\hline Aorta & $8.08 \pm 3.10$ & $10.31 \pm 4.13$ & $<0.001$ & $8.70 \pm 3.97$ & $11.60 \pm 5.53$ & $<0.001$ \\
\hline Portal vein & $8.00 \pm 2.62$ & $10.09 \pm 3.64$ & $<0.001$ & $4.17 \pm 2.84$ & $5.91 \pm 3.81$ & $<0.001$ \\
\hline
\end{tabular}

Table 4. Subjective image quality of $70-\mathrm{keV}$ virtual monoE images as compared to conventional $120-\mathrm{kVp}$ polychromatic images

\begin{tabular}{lccc}
\hline & Poly (median) & monoE (median) & $p$ value \\
\hline Noise & 3 & 4 & $<0.001$ \\
Soft tissue contrast & 3 & 4 & $<0.001$ \\
Sharpness & 3 & 4 & $<0.001$ \\
Overall image quality & 3 & 4 & $<0.001$ \\
\hline
\end{tabular}

Table 5. Inter-observer agreement (kappa scores) for subjective evaluation of $70-\mathrm{keV}$ virtual monoE images and conventional $120-\mathrm{kVp}$ polychromatic images

\begin{tabular}{lcc}
\hline & Poly & monoE \\
\hline Noise & 0.847 & 0.806 \\
Soft tissue contrast & 0.735 & 0.764 \\
Sharpness & 0.803 & 0.703 \\
Overall image quality & 0.767 & 0.762 \\
\hline
\end{tabular}

\section{Discussion}

In our prospective study, we compared the subjective and objective image quality of $70-\mathrm{keV}$ virtual monoE images to $120-\mathrm{kVp}$ standard polychromatic images obtained from the SDCT scanner. Virtual monoE images at 70-keV images provided significantly reduced objective image noise, increased SNR, and CNR. The qualitative scores for noise, soft tissue contrast, sharpness, and overall image quality were higher in $70-\mathrm{keV}$ images in comparison to conventional $120-\mathrm{kVp}$ polychromatic images.

Yamada et al. compared quantitative and subjective image quality of abdominal CT at $70-\mathrm{keV}$ virtual monoE images to conventional $120-\mathrm{kVp}$ polychromatic images from fast $\mathrm{kVp}$-switching dual-energy $\mathrm{CT}$ [2]. Filtered back projection (FBP) and iterative reconstruction (IR) were generated for both virtual monoE $70-\mathrm{keV}$ and polychromatic $120-\mathrm{kVp}$ images. They compared virtual monoE $70-\mathrm{keV}$ and polychromatic $120-\mathrm{kVp}$ images both subjectively and objectively. They concluded that $70-\mathrm{keV}$ virtual monoE images with FBP and IR have a higher image quality than polychromatic $120-\mathrm{kVp}$ images. The quality of $70-\mathrm{keV}$ images with IR was superior to $70-\mathrm{keV}$ images with FBP [2]. In our study, we compared the subjective and objective image quality of abdominal CT from the SDCT scanner, while in Yamada et al. study patients underwent sequential fast $\mathrm{kVp}$-switching dualenergy $(80 / 140 \mathrm{kVp})$ and single-energy $(120-\mathrm{kVp})$ abdominal enhanced CT. Furthermore, in our study we evaluated $70-\mathrm{keV}$ virtual monoE images with IR, not FBP. Our results were consistent with this mentioned clinical study.

Image quality of virtual monoE images at different levels of energy to conventional $120-\mathrm{kVp}$ in abdominal CT angiography from single-source dual-energy CT scan was compared in a study by Pinho et al. [14]. They evaluated the image quality in the same patients but images were obtained at different times with a 6-month inter-scan interval [14]. They concluded that $70-\mathrm{keV}$ virtual monoE images improve image quality for aortoiliac CT in comparison to conventional $120-\mathrm{kVp}$ images [14]. Their results were consistent with our results, but we compared just one level of energy $(70-\mathrm{keV})$ to conventional $120-\mathrm{kVp}$ at portal phase in intra-abdominal organs. In our study, both conventional $120-\mathrm{kVp}$ and $70-\mathrm{keV}$ virtual monoE images were created using single dataset obtained from single scan. However, their images were generated from two image sets obtained from two scans with six-month inter-scan interval. This can result in difference in noise or image quality, because of changes in the body weight or hemodynamics of the patients.

Matsumoto et al. compared the image quality of virtual monoE images from fast kilovoltage switching to conventional $120-\mathrm{kVp}$ in phantoms [15]. They concluded that $70-\mathrm{keV}$ virtual monoE images had lower noise and higher CNR in comparison to conventional $120-\mathrm{kVp}$ images [15]. Although they used phantoms and our study is a prospective study on patients, our results are consistent.

Based on our results and previous studies, $70-\mathrm{keV}$ virtual monoE images have lower noise and higher CNR and SNR in comparison to conventional $120-\mathrm{kVp}$ images. Since SDCT generates a dataset that provides conventional polychromatic images as well as retrospective reconstruction of different levels of virtual monoE images with several clinical applications as mentioned in Introduction, this new technology could have the potential to replace conventional CT technology. 
Our study had some limitations. Our subjective and objective assessment included different organs. However, same evaluation on different lesions and pathologies would be of great interest. All comparisons were made in the portal phase in our study. We also compared virtual monoE images at only one level of energy $(70-\mathrm{keV})$ to polychromatic $120-\mathrm{kVp}$ images. Further studies with different contrast phases (arterial, portal, and delayed) and multiple levels of virtual monoE images are warranted to confirm our findings.

\section{Conclusion}

Seventy-keV virtual monoE CT images for abdominal imaging can significantly improve the noise, SNR, and $\mathrm{CNR}$ as compared to conventional $120-\mathrm{kVp}$ polychromatic images. The noise, soft tissue contrast, sharpness, and overall image quality of virtual monoE images were much better than that of polychromatic images subjectively.

\section{Compliance with ethical standards}

Funding This study was funded by institutional research grant from Philips Healthcare.

Conflict of interest Prabhakar Rajiah received a speaker honorarium from Philips Healthcare. Amar Dhanantwari is an employee of Philips Healthcare. The other all authors declare that they have no conflict of interest.

Ethical approval All procedures performed in studies involving human participants were in accordance with the ethical standards of the institutional and/or national research committee and with the 1964 Helsinki declaration and its later amendments or comparable ethical standards.

Informed consent Informed consent was obtained from all individual participants included in the study.

\section{References}

1. Boone JM (2006) Multidetector CT: opportunities, challenges, and concerns associated with scanners with 64 or more detector rows. Radiology 241(2):334-337

2. Yamada Y, et al. (2014) Abdominal CT: an intra-individual comparison between virtual monochromatic spectral and polychromatic $120-\mathrm{kVp}$ images obtained during the same examination. Eur $\mathbf{J}$ Radiol 83(10):1715-1722

3. Chen AL, et al. (2015) Detection of gallbladder stones by dualenergy spectral computed tomography imaging. World J Gastroenterol 21(34):9993-9998

4. Karcaaltincaba M, Aktas A (2011) Dual-energy CT revisited with multidetector CT: review of principles and clinical applications. Diagn Interv Radiol 17(3):181-194

5. Remy-Jardin M, et al. (2014) Thoracic applications of dual energy. Semin Respir Crit Care Med 35(1):64-73

6. Kang MJ, et al. (2010) Dual-energy CT: clinical applications in various pulmonary diseases. Radiographics 30(3):685-698

7. Pomerantz SR, et al. (2013) Virtual monochromatic reconstruction of dual-energy unenhanced head CT at $65-75 \mathrm{keV}$ maximizes image quality compared with conventional polychromatic CT. Radiology 266(1):318-325

8. Lee SH, et al. (2013) Additional value of dual-energy CT to differentiate between benign and malignant mediastinal tumors: an initial experience. Eur J Radiol 82(11):2043-2049

9. Vliegenthart R, et al. (2012) Dual-energy CT of the heart. AJR Am J Roentgenol 199(5 Suppl):S54-S63

10. Kulkarni NM, et al. (2013) Determination of renal stone composition in phantom and patients using single-source dual-energy computed tomography. J Comput Assist Tomogr 37(1):37-45

11. Nicolaou S, et al. (2012) Dual-energy CT: a promising new technique for assessment of the musculoskeletal system. AJR Am J Roentgenol 199(5 Suppl):S78-S86

12. Zhao LQ, et al. (2012) Improving image quality in portal venography with spectral CT imaging. Eur J Radiol 81(8):1677-1681

13. Kalra MK, et al. (2003) Low-dose CT of the abdomen: evaluation of image improvement with use of noise reduction filters pilot study. Radiology 228(1):251-256

14. Pinho DF, et al. (2013) Initial experience with single-source dualenergy CT abdominal angiography and comparison with singleenergy CT angiography: image quality, enhancement, diagnosis and radiation dose. Eur Radiol 23(2):351-359

15. Matsumoto K, et al. (2011) Virtual monochromatic spectral imaging with fast kilovoltage switching: improved image quality as compared with that obtained with conventional $120-\mathrm{kVp}$ CT. Radiology 259(1):257-262 